\title{
Untersuchungen an gangliosidartigen Substanzen aus menschlicher Milz
}

Dem Gedächtnis von Prof. Dr. S. J. Thannhauser gewidmet, der am 28. 6. 196580 Jahre alt geworden wäre.

\author{
Von A. WAGNER und H. WEICKER
}

Aus der Abteilung für Stoffwecbselforschung (Leiter: Prof. Dr. H. Weicker) der Mediziniscben Universitäts-Poliklinik Heidelberg (Direktor: Prof. Dr. H. Plügge)

(Eingegangen am 28. Juli 1965)

\begin{abstract}
Nach einer Modifikation der von FolCH und Mitarbeitern angegebenen Methode zur Gangliosidpräparation wurden aus weitgehend von Erythrozyten befreitem Milzgewebe des Menschen eine gangliosidartige Substanz in einer Ausbeute von $0,06 \%$ der Trockensubstanz gewonnen. Im Gegensatz zu den Hirngangliosiden, die als Fettsäure fast ausschließlich Stearinsäure enthalten, fand sich hier vorwiegend Nervon-, Lignocerin- und Behensäure. Chromatographisch erwies sich das Milzgangliosid als inhomogen. Von den 6 gefundenen Fraktionen überwiegt quantitativ eine schnellwandernde hexosaminfreie Fraktion, die aus Sphingosin, einer Fettsäure, Galaktose, Glucose und Neuraminsäure im molaren Verhältnis von 1:1:1:1:1 aufgebaut ist. Sie ist damit identisch mit den von YAMAKAWA und SuzUKr und von KLENK und Mitarbeitern aus Pferde- bzw. Hundeerythrozyten dargestellten Hämatosiden. Von den anderen Fraktionen ist $M_{5}$ wahrscheinlich hexosaminfrei, die Fraktionen $M_{G_{1}}$ bis $M_{4} G_{4}$ enthalten im Gegensatz zu den Hirngangliosiden sowohl Galaktosamin als auch Glucosamin. Sie unterscheiden sich durch das molare Verhältnis von Galaktose und Glucose, das in $\mathrm{MG}_{4} 1: 1$, in den langsamer wandernden Fraktionen 2:1 beträgt.
\end{abstract}

Using a modification of the method of Folch et al. for the preparation of gangliosides, a substance resembling a ganglioside was isolated from human spleen, which had been largely freed from erythrocytes. The yield was $0.06 \%$ of the dry weight of starting material. Unlike the cerebral gangliosides, which contain almost exclusively stearic acid, the predominant acids were nervonic, lignoceric and behenic. The spleen ganglioside was not chromatographically homogeneous. Six fractions were shown; the predominant one migrated rapidly, was free from hexoseamine and contained sphingosine, a fatty acid, galactose, glucose and neuramic acid in the molar tations 1:1:1:1:1. It is thus identical with the haematoside prepared by YAMAKAwA and SUzUKI and by KLENK et al. from horse and dog erythrocytes. Amongst the other fractions, $M_{G_{5}}$ is probably free from hexoseamine, while fractions $M_{1} G_{1}$ to $\mathbf{M G}_{4}$, unlike the brain gangliosides, contain both galactoseamine and glucoseamine. They differ from one another in their molar ratio of galactose and glucose, which 1:1 in $\mathbf{M G}_{4}$ and 2:1 in the slower fractions.

Bei den von KLENK (1) erstmals aus der grauen Rinde des Gehirns isolierten Gangliosiden handelt es sich um Glykolipide, die sich aus folgenden Elementen aufbauen: Sphingosin, Stearinsäure, Galaktose, Glucose, Galaktosamin und N-acetyl-Neuraminsäure. Ähnliche Substanzen wurden seitdem in den Erythrozyten verschiedener Tierarten z. B. Rind, Pferd, Hund u. a wie auch im menschlichen Erythrocytenstroma gefunden. Nach den Untersuchungen von WALZ (2) sowie LEVENE und Landsteiner (3), die aus der Protagonfraktion der Milz und Niere vom Rind bzw. Pferd eine stark huminbildende Substanz bekamen, war auch hier mit dem Vorliegen solcher Glykolipide zu rechnen. Der Nachweis gelang KLENK und RENNKAMp 1942 (4). Sie isolierten aus Rindermilz ein Gangliosid mit einem Kohlenhydratgehalt von $39,4 \%$. Im Gegensatz zu den Hirngangliosiden, die fast ausschließlich Stearinsäure enthalten, wurden als Fettsäurekomponenten des Milzgangliosids Behen- und Lignocerinsäure ermittelt. SVENNERHOLM schließlich berichtete 1963 über eine aus Menschenmilz isolierte Gangliosidfraktion mit 28,1\% Hexosen; $24 \%$ $\mathrm{N}$-acetyl-Neuraminsäure und 0,6\% Hexosamin (5). 1962 hatten wir unter der Anleitung von Herrn Prof. S. J. ThanNhauser gleichfalls mit der Isolierung gangliosidartiger Substanzen aus menschlicher Milz begonnen. Utber die Untersuchungsergebnisse soll im folgenden berichtet werden.

\section{Methodik}

Zur Untersuchung kamen makroskopisch normale menschliche Milzen. Hämoblastosen und andere Erkrankungen mit Splenomegalie wurden nicbt einbezogen. Kapsel und Gefäß wurden entfernt, das Milzgewebe dann in möglichst feine Schnitte geschnitten und diese zur weitgehenden Elimination der Erythrozyten zwischen verschiedene Lagen von Filterpapier gepreßt. Das Filterpapier wurde $3 \mathrm{mal}$ gewechselt. Die Gewebsschnitte hatten danach eine graurote Farbe. Sie wurden im Multimix zerkleinert und mit einem Gemisch von Aceton und physiologischer Kochsalzlösung (1:1) so oft gewaschen, bis das Filtrat farblos blieb. Der Gewebebrei wurde dann mit Aceton getrocknet, mit etwa dem fünffachen Volumen $3 \mathrm{mal}$ für 30 Minuten mit kochendem Methanol extrahiert und heiß filtriert. Das Präzipitat, das sich nach 18 stündigem Stehen bei $+4^{0}$ gebildet hatte, wurde abfiltriert, in Chloroform : Methanol (2:1) aufgenommen und entsprechend der von Foucr und Mitarbeitern (6) sowie von Bogoch (7) angegebenen Methode die Ganglioside aus der wäßrigen Phase eines ChloroformMethanol-Wassergemisches nach Dialyse und Gefriertrocknung gewonnen.

$\mathrm{Da}$ dünnschichtchromatographische Kontrollen ergeben hatten, daß sowohl in dem zur Extraktion benützten Methanol wie auch in der zur Reinigung der Ganglioside benützten organischen Phase noch relativ 
viel gangliosidartige Substanzen vorhanden waren, wurden diese im Rotationsverdampfer stark eingeengt. Durch Abkühlen in einem Acetonbad von $-70^{\circ}$ wurden hieraus noch reichlich Lipide ausgeschieden, die dem gleichen Reinigungsverfahren, wie oben beschrieben, unterworfen wurden.

Dïnnscbicbt- und Säulenclbromatograpbie: Kieselgel G; Laufmittel: Chloroform: Methanol: $\mathrm{H}_{2} \mathrm{O}(61: 32: 7)(20,21)$; Sprühreagens: Orcinolspray ( $10 \mathrm{~m} l$ 1,6-proz. wäßrige Orcinollösung $+75 \mathrm{ml}$ 60-proz. $\mathrm{H}_{2} \mathrm{SO}_{4}$ ); 20 Min. Trockenschrank bei $40^{\circ}$.

Spbingosinbestimmung: Qualitativ als Methylsphingosin papierchromatographisch descendierend auf Whatmanpapier No. I mit Butanol:Eisessig: $\mathrm{H}_{2} \mathrm{O}(12: 3: 5)$ für 16 Stdn.; dünnschichtchromatographisch auf Kieselgel $G$ mit Methanol und auf silikonisierten Kieselgelplatten mit Acetonitril : Eisessig : $\mathrm{H}_{2} \mathrm{O}$ $(70: 10: 25)$. - Ninhydrinspray: Quantitativ nach KIETA und SCHMIDT (8).

Fettsäıreanalyse: Qualitativ: gaschromatographisch; quantitativ: Hydroxamsäuremethode nach SNYDER (9).

Hexosen und Hexosamine: Qualitativ: Nach Hydrolyse in $3 n \mathrm{HCl}$ für 90 Min. papierchromatographisch auf Whatman No. I descendierend für 16 Stdn. mit Pyridin: Butanol: $\mathrm{H}_{2} \mathrm{O}$ (4:6:3) - Silbernitrat.

Quantitativ: a) Cysteinreaktion nach Dische (10). b) Bestimmung der Gesamtreduktion im Hydrolysat nach NeLson (11) und der Glucose enzymatisch mit Glucoseoxydase. c) Nach papierchromatographischer Trennung Ausschneiden der durch Referenzen markierten Zuckerfraktionen und Bestimmung der Reduktion des Eluates. d) Hexosamin nach Elson und Morgan (12).

Neuraminsäure: nach SvenNerholm (13).

\section{Ergebnisse}

Aus $963 \mathrm{~g}$ acetongetrockneten Milzgewebes erhielten wir nach Gefriertrocknung $192 \mathrm{mg}$ einer gangliosidartigen Substanz in Form eines weißen voluminösen Pulvers. Es war in Wasser (unter Bildung einer opaleszenten Flüssigkeit) wie in Chloroform:Methanol $(2: 1)$ sehr gut löslich. Beim Erhitzen auf $215^{\circ}$ sinterte die Substanz zusammen und färbte sich schwarz. Die Phosphorbestimmung von $0,21 \%$ weist allerdings noch auf eine Verunreinigung mit Phospholipiden hin.

Bausteinanalyse: Nach Hydrolyse mit 10-proz. methanolischer $\mathrm{HCl}$ und Extraktion der Fettsäuremethylester in Petroläther wurden gaschromatographisch folgende Fettsäuren identifiziert: Palmitinsäure $(8,8 \%)$, Stearinsäure (13,2\%), Arachidonsäure (4,5\%), Nervonsäure $(25,7 \%)$, Behensäure $(24,9 \%)$ und Lignoceöinsäure (21,9\%). Der methanolische Rückstand wurde mit $\mathrm{KOH}$ neutralisiert und mit Chloroform extrahiert. Chromatographisch wurde ein ninhydrinpositiver Fleck gefunden, der auf 3 verschiedenen Trennmedien und in 3 verschiedenen Lösungsmittelsystemen den gleichen $R_{\mathrm{F}-}$ Wert aufwies wie in gleicher Weise präpariertes Methylsphingosin aus Cerebrosiden. Spuren einer weiteren ninhydrinpositiven Substanz wurden nicht identifiziert. Die Ergebnisse der qualitativen und quantitativen Zuckeranalyse dieser wasserlöslichen Glykolipide aus menschlicher Milz werden in Abbildung $1 \mathrm{bzw}$. Tabelle 1 wiedergegeben.

Bei der Dünnschichtchromatographie trennte sich das Präparat in 6 Fraktionen auf (Abb. 2). Bei dem Versuch,

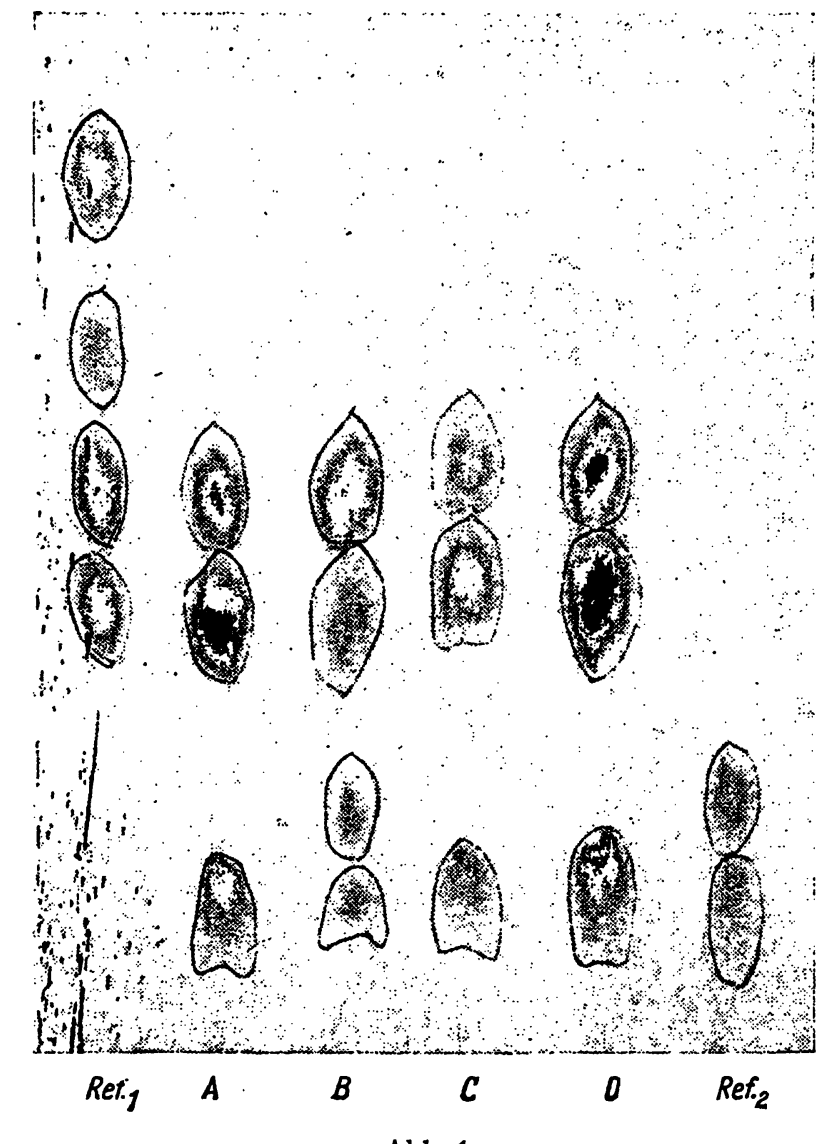

Abb. 1

Papierchromatogramm der Zuckerkomponenten von hydrolysierten Gangliosiden aus menschlicher Milz (B) im Vergleich zu menschlichem Gehirn (A) und Milz und Gehirn vom Rind (Cu. D) Referenzzucker, jeweils von oben nach unten: 1: Fucose, Mannose, Glucose, Galaktose; 2: Glucosamin, Galaktosamin.

Tab. 1

Kohlenhydrat-Analyse der wasserlöslichen Glykolipide aus menschlicher Milz

\begin{tabular}{lr}
\hline$\cdots \quad$ Galaktose $^{1}$ ) & $12,2 \%$ \\
Glucose $^{1}$ ) & $10,6 \%$ \\
Hexosen $^{1}$ ) Gesamt & $22,8 \%$ \\
Hexosen (Anthron-Methode) & $25,0 \%$ \\
Hexosamine & $1,2 \%$ \\
N-acetyl-Neuraminsäure & $16,4 \%$ \\
Kohlenhydrate Gesamt & $40,4 \%$ \\
\hline
\end{tabular}

1) Cysteinreaktion nach Drsche (10)

diese Fraktionen säulenchromatographisch zu isolieren, zeigte es sich, daß die quantitativ überwiegende Fraktion $\mathrm{MG}_{6}$ sich nochmals in 2 Untergruppen auftreninte (Abb. 3). Eine Rechromatographie ergab keine saubere Isolierung dieser Komponenten, so daß sie zur Vermeidung weiterer Substanzverluste gemeinsam als Fraktion $\mathrm{MG}_{6}$ untersucht wurden.

Die geringen $R_{\mathrm{F}}$-Wert-Differenzen und die verhältnismäßig geringe Quantität der übrigen Fraktionen hatte zur Folge, daß bei ihrer Isolierung gewisse Uberlappungen in Kauf genommen werden mußten (Abb.3), die Fraktionen also nicht ideal sauber waren. 


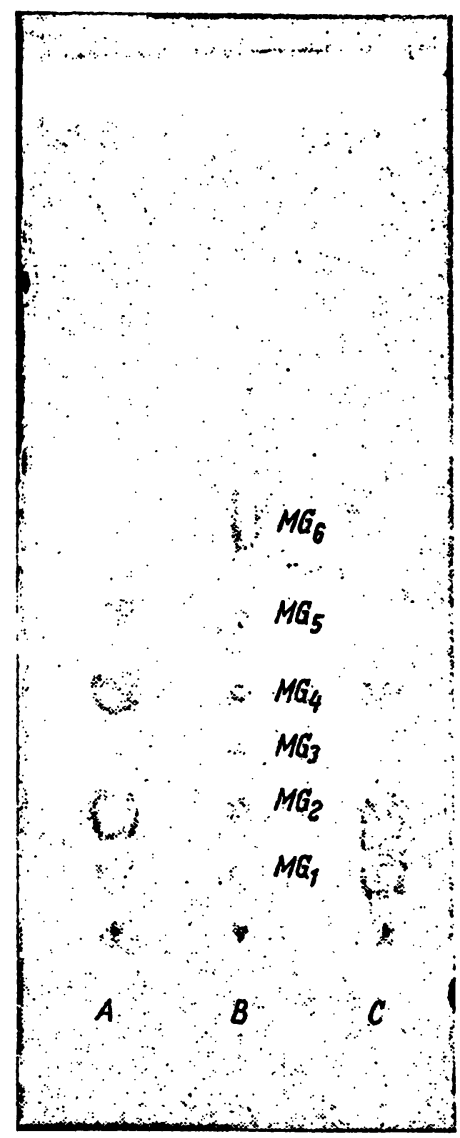

Abb. 2

Dünnschichtchromatogramm der Ganglioside aus menschlicher Milz (B) im Vergleich zu Milzgangliosiden vom Rind (A) und Hirngangliosiden vom Menschen (C)
$(3)$

(i)
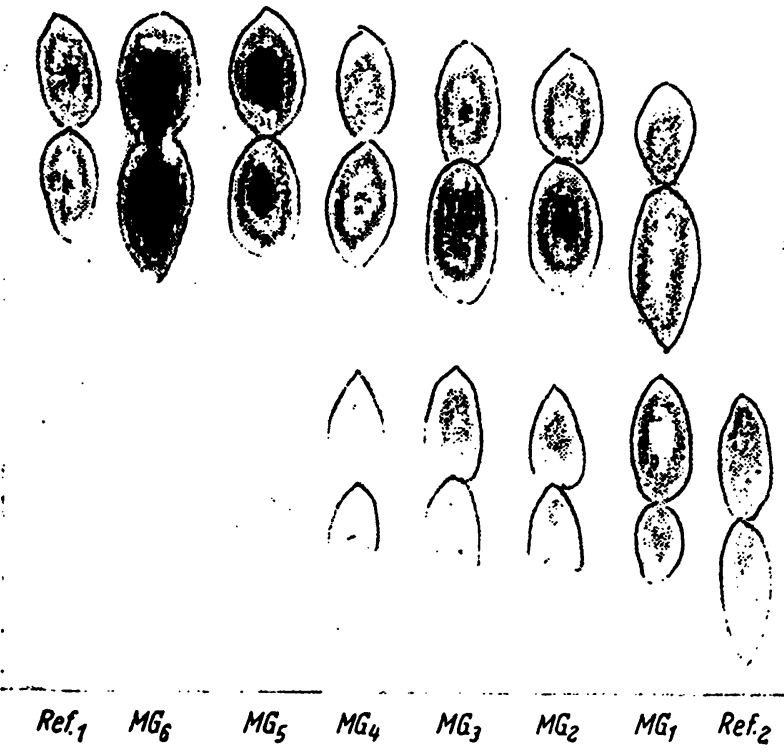

Abb. 4

Papierchromatographische Zuckeranalyse der hydrolysierten Milzgangliosidfraktionen $\mathrm{MG}_{1}-\mathrm{MG}_{6}$.

Referenzzucker, jeweils von oben nach unten: 1 : Fucose, Mannose, Glucose, Galaktose; 2: Glucosamin, Galaktosamin.

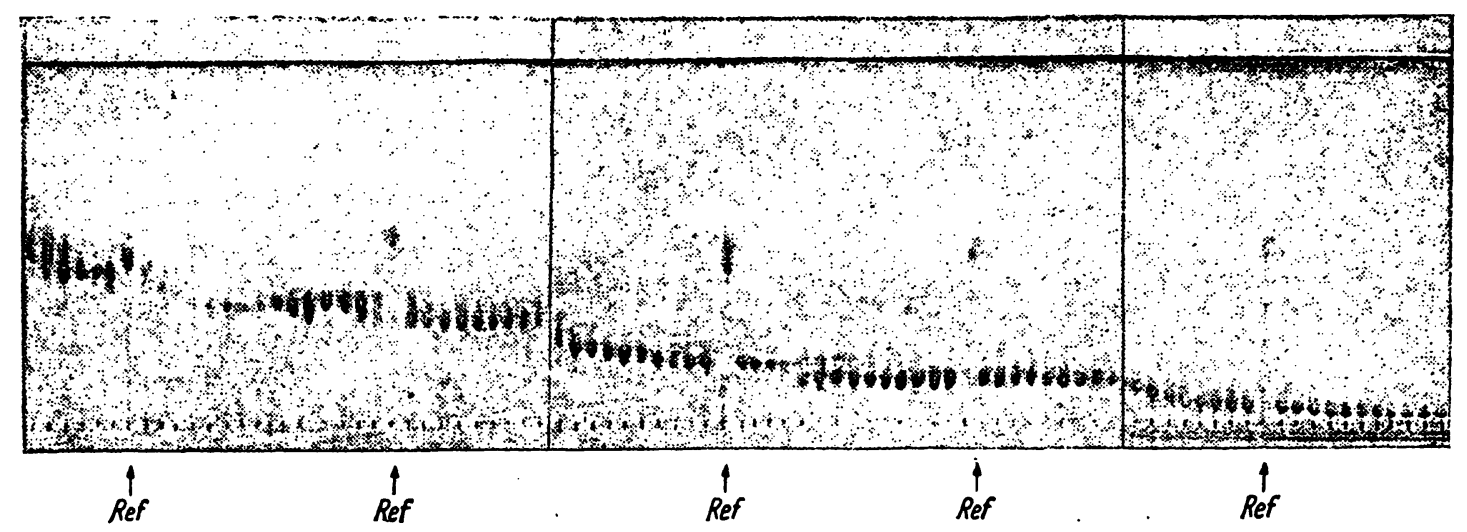

Abb. 3

Dünnschichtchromatographische Kontrolle der säulenchromatographisch isolierten Fraktionen von Milzgangliosiden.

Von den $158,5 \mathrm{mg}$ des zur Chromatographie gelangten Gangliosidgemisches entfielen $60,5 \mathrm{mg}$ bzw. $38 \%$ auf die schnellwandernde Fraktion $\mathrm{MG}_{6}$, der Rest auf die 5 anderen Fraktionen, von denen $\mathrm{MG}_{3}$ den geringsten Anteil stellte. Die Bausteinanalyse ergab in allen 6 Fraktionen einen Aminoalkohol mit dem gleichen $R_{F}$-Wert wie Sphingosin. Die prozentuale Verteilung der Fettsäuren in diesen 6 Fraktionen ist in Tabelle 2 zusammengestellt. Bei der qualitativen Zuckeranalyse (s. Abb. 4) waren in den Fraktionen $\mathrm{MG}_{1}-\mathrm{MG}_{4}$ Galaktose, Glu-
Tab. 2

Prozentuale Verteilung der Fettsäuremethylester in den säulenchromatographisch isolierten Milzgangliosidfraktionen

\begin{tabular}{lrllllll}
\hline & $\mathrm{C}_{16}$ & $\mathrm{C}_{18}$ & $\mathrm{C}_{20}$ & $\mathrm{C}_{22}$ & $\mathrm{C}_{23}$ ? & $\mathrm{C}_{24}$ & $\mathrm{C}_{24}=$ \\
\hline $\mathrm{MG}_{6}$ & 10,4 & 6,5 & 7,7 & 32,4 & 11,2 & 6,6 & 27,0 \\
$\mathrm{MG}_{5}$ & 5,6 & 22,0 & 3,2 & 21,9 & 6,0 & 18,0 & 25,9 \\
$\mathrm{MG}_{4}$ & 7,8 & 24,3 & 3,2 & 16,8 & 4,5 & 15,5 & 28,2 \\
$\mathrm{MG}_{3}$ & 6,0 & 24,3 & 2,9 & 21,3 & 3,1 & 12,2 & 30,1 \\
$\mathrm{MG}_{2}$ & 6,5 & 41,9 & 5,6 & 16,7 & 1,8 & 9,5 & 18,1 \\
$\mathrm{MG}_{1}$ & 10,3 & 26,5 & 5,1 & 22,6 & 2,0 & 11,2 & 22,5 \\
\hline
\end{tabular}


cose, Galaktosamin und Glucosamin nachweisbar. Die geringe Anfärbung im Galaktosaminbereich bei $\mathrm{MG}_{5}$ könnte evtl. auf geringe Verunreinigungen mit $\mathrm{MG}_{4}$ zurückzuführen sein. $M G_{6}$ ist sicher frei von Aminozuckern. Die quantitativen Zuckerbestimmungen für die Fraktionen $M G_{1}-M_{5}$ sind in Tabelle 3 wiedergegeben. Infolge der geringen zur Verfügung stehenden Menge wurden für die Hexosen nur die Cysteinreaktionen durchgeführt. Alle Ergebnisse resultieren aus einer einmaligen Bestimmung, haben daher nur einen bedingten Aussagewert. Zur Sicherung der molaren Relationen haben wir daher die Hydrolysate der Fraktionen chromatographiert, die Flecken ausgeschnitten, eluiert und die Reduktion mit der Nelson-Methode bestimmt. Die Ergebnisse sind aus Tabelle $4 \mathrm{zu}$ ersehen. Von der Fraktion $\mathrm{MG}_{6}$ lag genügend Substanz für gründlichere Analysen vor. Durch die Rechromatographie lag der Phosphorgehalt dieser Fraktion mit $0,13 \%$ auch niedriger, d. h. sie war sauberer als das Ausgangsgemisch. Tabelle 5 enthält die Ergebnisse der quantitativen Bestimmung sowie die molațen Relationen.

Tab. 3

Kohlenhydratanalyse der Milzgangliosidfraktionen $M G_{1}$ bis $M_{5}$ (Werte in \%)

\begin{tabular}{lccccc}
\hline & Galaktose & Glucose & Hexosen & $\begin{array}{c}\text { Hexos- } \\
\text { amine }\end{array}$ & $\begin{array}{c}\text { N-acetyl- } \\
\text { Neuramin- } \\
\text { säure }\end{array}$ \\
\hline $\mathrm{MG}_{1}$ & 15,6 & 7,2 & 22,8 & 11,0 & 11,2 \\
$\mathrm{MG}_{2}$ & 16,3 & 6,5 & 22,8 & 11,6 & 12,8 \\
$\mathrm{MG}_{3}$ & 15,4 & 7,5 & 22,9 & 10,8 & 12,4 \\
$\mathrm{MG}_{4}$ & 14,1 & 6,5 & 20,6 & 12,0 & 12,8 \\
$\mathrm{MG}_{5}$ & 8,3 & 9,3 & 17,6 & 0,6 & 16,8 \\
\hline
\end{tabular}

Tab. 4

Molares Verhältnis der Hexosen und Hexosamine aus den säulenchromatographisch isolierten Milzgangliosiden (s. Text)

\begin{tabular}{cccccccc}
\hline & Galaktose & & Glucose & \multicolumn{2}{c}{ Galaktosamin } & Glucosamin \\
\hline $\mathrm{MG}_{1}$ & 2 & $:$ & 0,84 & $:$ & 0,72 & $:$ & 1,1 \\
$\mathrm{MG}_{2}$ & 2 & $:$ & 0,97 & $:$ & 0,82 & $:$ & 0,86 \\
$\mathrm{MG}_{3}$ & 2 & $:$ & 0,91 & $:$ & 0,81 & $:$ & 0,92 \\
$\mathrm{MG}_{4}$ & 1 & $:$ & 0,72 & $:$ & 0,79 & $:$ & 0,82 \\
$\mathrm{MG}_{5}$ & 1 & $:$ & 1,16 & $:$ & $?$ & $:$ & - \\
\hline
\end{tabular}

Tab. 5

Analytische Daten der Milzgangliosidfraktion $\mathrm{MG}_{6}$

\begin{tabular}{|c|c|c|c|c|}
\hline Galaktose & Glucose & $\begin{array}{c}\mathrm{N} \text {-acetyl- } \\
\text { neuraminșäure }\end{array}$ & $e^{\text {Sphingosin }}$ & Fettsäuren \\
\hline $\begin{array}{l}\left.14,6 \%{ }^{1}\right) \\
\left.12,6 \%{ }^{2}\right)\end{array}$ & $\begin{array}{l}\left.14,0 \%{ }^{1}\right) \\
\left.11,0 \%^{2}\right)\end{array}$ & $19,9 \%$ & $24,8 \%$ & $25,0 \%$ \\
\hline \multicolumn{5}{|c|}{ Molares Verbältnis } \\
\hline 1 & 0,87 & 0,95 & 1,15 & 1,08 \\
\hline Elementaranalyse & C & $\mathrm{H}$ & $\mathrm{N}$ & \\
\hline $\begin{array}{l}\text { Gefunden: } \\
\text { Berechnet: }\end{array}$ & $\begin{array}{l}59,1 \% \\
60,6 \%\end{array}$ & $\begin{array}{l}9,83 \% \\
9,8 \%\end{array}$ & $\begin{array}{l}2,98 \% \\
2,3 \%\end{array}$ & \\
\hline
\end{tabular}

1) Bestimmung mit Cysteinreaktion nach Dische (10).

2) Nach Hydrolyse Gesamtreduktion nach Nelson (11); Glucose mit Glucoseoxydase bestimmt.

\section{Diskussion}

Wie aus Rindermilz lassen sich auch aus Menschenmilz wasserlösliche neuraminsäurehaltige Glykolipide, also gangliosidartige Substanzen gewinnen. Die effektive Ausbeute liegt mit 0,03\% des getrockneten Milzgewebes erwartungsgemäß weit unter dem Gangliosidgehalt des menschlichen Gehirns. Hier kann er bis zu $1 \%$ der Trockensubstanz betragen. Verluste während der Milzgangliosidpräparation sind zu berücksichtigen. Aus dem Verhältnis des Neuraminsäuregehaltes des ersten Extraktes zu dem des analysierten Präparates würde sich ein Gangliosidanteil von $0,06 \%$ der Trockensubstanz errechnen. Diese Milzganglioside unterscheiden sich von den Hirngangliosiden qualitativ sowohl in ihrer Fettsäure- wie auch der Kohlenhydratzusammensetzung. Während in den Hirngangliosiden fast ausnahmslos Stearinsäure gefunden wird, liegen in den Milzgangliosiden vor allem gesättigte und ungesättigte $\mathrm{C}_{22^{-}}$und $\mathrm{C}_{24}$-Fettsäuren vor. Bei der Zuckeranalyse wurde neben den in Hirngangliosiden vorkommenden Substanzen Neuraminsäure, Galaktose, Glucose und Galaktosamin auch Glucosamin gefunden. Interessanterweise ist in dem mit gleicher Methode aus Rindermilz gewonnenen Gangliosid kein Glucosamin nachweisbar. An den quantitativen Untersuchungen ist der relativ niedrige Kohlenhydratgehalt der Milzganglioside gegenüber den Hirngangliosiden bemerkenswert. Dieser relativ niedrige Kohlenhydratgehalt der Milzganglioside sowie die langkettigen Fettsäuren dürften die Ursache für ihre größere Affinität zu organischen Lösungsmitteln sein. Eine Modifikation der Gangliosidpräpäration ist daher erforderlich. Der auffallend niedrige Hexosamingehalt findet seine Erklärung darin, daß sich das Gangliosidpräparat chromatographisch als inhomogen erwies. Von den 6 erfaßbaren Fraktionen dominiert quantitativ eine relativ schnell wandernde, die hexosaminfrei ist. Die Analyse der 5 langsamer wandernden Fraktionen ist wegen der gèringen Quantität und der kleinen $R_{\mathrm{F}}$-Differenzen, die weitgehende Überlappungen bei der Säulenfraktionierung verursachten, leider etwas unbefriedigend geblieben. Mit entsprechender Vorsicht darf gesagt werden, daß Spbingosin in allen Fraktionen nachweisbar ist. Die Fettsäuren scheinen für die Fraktionierung in diese 5 Gruppen nicht von wesentlicher Bedeutung zu sein, d. h. wenn eine Fettsäure ausschließlich oder überwiegend in einer der Fraktionen vorkäme, müßten trotz bestehender Fraktionsüberschneidungen bei der gaschromatographischen Untersuchung bedeutendere Verschiebungen des Fettsäurespektrums zustande kommen als sie hier gefunden wurden. Die Tabelle 2, in der diese Ergebnisse niedergelegt sind, läßt zwar erkennen, daß in den langsamer wandernden Fraktionen $M G_{1}$ und $\mathrm{MG}_{2}$ Stearin- und Palmitinsäure überwiegen, während in den schnellwandernden Fraktionen Nervonsäure, Lignocerin- und Behensäure mehr in den Vordergrund treten. Wie weit dies eine Eigentümlichkeit der Gangliosidfraktionen ist oder ob hierbei die SphingomyelinVerunreinigungen mitwirken, vermögen wir nicht zu entscheiden. 
Bezüglich der Koblenbydratkomponenten ergaben die quantitativen Bestimmungen und vor allem die Untersuchungen nach Elution der chromatographisch getrennten Zucker folgende Befunde: In den langsam wandernden Fraktionen $M_{1,2}$ und 3 dürfte das molare Verhältnis von Galaktose und Glucose $2: 1$, in den schneller wandernden Fraktionen $\mathrm{MG}_{4}$ und 5 dagegen 1:1 betragen. Von den Aminozuckern waren in den Fraktionen $\mathrm{MG}_{1}$ bis $\mathrm{MG}_{4}$ sowohl Galaktosamin als auch Glucosamin nachweisbar. Der geringe Hexosaminfleck in $\mathrm{MG}_{5}$ stammt wahrscheinlich aus Verunreinigungen mit Fraktion $\mathrm{MG}_{4}$. Ein molares Verhältnis der Aminozucker zur Glucose von 1:1 wird nach der Elution wahrscheinlich. Die quantitative Neuraminsäurebestimmung in den langsam wandernden Fraktionen erscheint fragwürdig und läßt uns von einer Bestimmung der molaren Relation absehen. Wahrscheinlich sind diese Fraktionen aber nicht so neuraminsäurereich wie die entsprechenden Fraktionen aus Hirngangliosiden.

Sichere Aussagen sind über die quantitativ dominierende Fraktion $\mathrm{MG}_{6}$ möglich. Sie enthält Sphingosin, eine Fettsäure, Galaktose, Glucose und N-Acetylneuraminsäure im äquimolaren Verhältnis. Bei den Fettsäuren handelt es sich vorwiegend um Nervon-, Lignocerin- und Behensäure. Im Dünnschichtchromatogramm war diese Fraktion immer als singulärer, allerdings flammig ausgezogener Fleck dargestellt. Bei der säulenchromatographischen Trennung ergaben sich 2 Unterfraktionen. Wir haben von der Dünnschichtplatte einige Flecken der oberen und unteren Subfraktion eluiert und hydrolysiert. Nach der chromatographischen Trennung sind die Zucker nicht verschieden. Für diese Subfraktionierung sind wahrscheinlich die verschiedenen Fettsäuren verantwortlich. Interessant erscheint an dieser Milzgangliosidfraktion $\left(\mathrm{MG}_{6}\right)$, daß sie offenbar identisch ist mit den von Yamakawa und SuzukI $(14,15)$ sowie KLENK und Mitarbeitern $(16,17)$ aus Pferdeerythrozytenstroma isolierten Hämatosiden bzw. den von KLENK und Heuer (18) aus Hundeerythrozyten dargestellten Gangliosiden. Mit Sicherheit kann diese Fraktion aber nicht aus Menschenerythrozyten stammen, die evtl. trotz der beschriebenen Säuberung noch im Milzgewebe verblieben waren, da die von KLENK und Mitarbeitern $(16,19)$ aus Menschenerythrozyten gewonnenen Glykolipide (Globoside) galaktosaminhaltig und neuraminsäurefrei sind.

\section{Literatur}

1. KLENK, E., Hoppe-Seyler's Z. physiol. Chem. 235, 24 (1935). 2. Walz, E., Hoppe-Seyler's Z. physiol. Chem. 166, 210 (1957). 3. Levene, D. A. und K. Landsteiner, J. biol. Chemistry 75, 607 (1927). - 4. KLenK, E. und F. Rennkamp, Hoppe-Seyler's Z. physiol. Chem. 272, 253 (1942). - 5. Svennerholm, L., Acta chem. scand. 17, 860 (1963). - 6. Forch, J., S. Arsove und J. A. Meath, J. biol. Chemistry 191, 819 (1951). - 7. Bogoch, S., Biochem. J. 68, 319 (1958). - 8. KretA, A. und G. Schmidt, noch nicht veröffentlicht. - 9. SNYDER, F. und N. STEPHENS, Biochim. Biophysica Acta (Amsterdam) 34, 244 (1959). - 10. Dische, Z., in: Methods of Biochemical Analysis, herausgeg. von D. Glick, Interscience, New York (1955). Bd. II, S. 313 - 11. Nelson, N., J. Biol. Chemistry 153, 375 (1944). - 12. Elson, L. A. und W. T. J.
Morgan, Biochem. J. 27, 1824 (1933). - 13. Svennerholm, L., Acta Soc. med. Upsaliensis 61, 287 (1956). - 14. Yamakawa, T. und S. Suzukr, J. Biochemistry (Tokyo) 38, 199 (1951). - 15. Yamakawa, T. und S. Suzukr, J. Biochemistry (Tokyo) 39, 393 (1952). - 16. Klenk, E. und H. Wolter, Hoppe-Seyler's Z. physiol. Chem. 291, 259 (1952). - 17. KLeNK, E. und K. LaUENSTEIN, Hoppe-Seyler's Z. physiol. Chem. 295, 164 (1953). - 18. KLENK, E. und K. Heuer, Dtsch. Zschr. Verdauungskrkh. 20, 180 (1960). - 19. Klenk, E. und K. Lauenstein, Hoppe-Seyler's $Z$. physiol. Chem. 291, 249 (1952). - 20. DaIN, J., E. Willis, E. I. Sweet, G. Schmidt und S. J. Thannhauser, Federat. Proc. 21, 282 (1962). - 21. WAGNER, H., L. Hörhammer und P. WolfF, Biochem. Z. 175 (1961).
Dr. med. A. Wagner

Med. Universitäts-Poliklinik 69 Heidelberg, Hospitalstr. 3 